\title{
ON THE SOLUTIONS OF CLAMPED REISSNER-MINDLIN PLATES UNDER TRANSVERSE LOADS*
}

\author{
BY \\ THOMAS C. ASSIFF ( EDS CAD/CAM Department, Southfield, MI) \\ AND \\ DAVID H. Y. YEN ( Michigan State University, East Lansing, MI)
}

\begin{abstract}
The governing equations in the Reissner-Mindlin theory may be written in a form such that a small parameter $\varepsilon$ is involved. This parameter $\varepsilon$ depends on a combination of the shear modulus and the plate thickness. The governing equations are singularly perturbed with respect to $\varepsilon$. However, as $\varepsilon \rightarrow 0$ one does recover the biharmonic equation of the classical plate theory. In a previous work of ours [1] the behavior of solutions for clamped Reissner-Mindlin plates as $\varepsilon \rightarrow 0$ was studied and it was shown there that these solutions tend continuously, in various functional norms, to their corresponding solutions in the classical plate theory. This paper deals with two specific questions concerning the detailed dependence of these solutions on $\varepsilon$ as $\varepsilon \rightarrow 0$. We shall show the nonexistence of regular asymptotic expansions of the solutions in integral powers of $\varepsilon$ for general clamped Reissner-Mindlin plates. We shall also construct an exact solution for a circular plate which exhibits dependence on fractional powers of $\varepsilon$. This latter solution shows a boundary layer phenomenon, decaying away from the boundary, often encountered in singular perturbation problems.
\end{abstract}

1. Introduction. We consider a clamped plate of uniform thickness and constant material properties occupying a closed, bounded, and connected region $\bar{\Omega}$ of the $x y$-plane. We denote the interior of the region by $\Omega$ and its boundary by $\partial \Omega$, which is assumed sufficiently smooth or polygonal.

In [1] the governing equations in the Reissner-Mindlin plate theory $[2,3]$ have been written in the form

$$
\left.\begin{array}{rl}
\frac{1}{2}\left[(1-\mu) \nabla^{2} \psi-(1+\mu) \nabla(\nabla \cdot \psi)\right]-\varepsilon^{-1}(\psi+\nabla w) & =0 \\
\varepsilon^{-1}\left(\nabla^{2} w+\nabla \cdot \psi\right) & =\frac{p}{D}
\end{array}\right\} \text { in } D
$$

*Received June 24, 1986. 
where $w$ is the transverse deflection, $\psi=\left(\psi_{x}, \psi_{y}\right)$ is the vector whose components are the (negative of, for convenience) plate rotations in the directions of the coordinate axes, $\mu$ is Poisson's ratio, $p$ is the transverse load, and $D$ is the plate modulus. $\varepsilon$ above is a parameter defined by

$$
\varepsilon=\frac{h_{0}^{2}}{6(1-\mu) \kappa^{2}},
$$

where $h_{0}$ is the plate thickness and $\kappa^{2}$ is a constant introduced by Mindlin [3] in the definition of the "modified" shear modulus $G^{\prime}=\kappa^{2} G$.

To equations (1.1) we add the clamped edge boundary conditions

$$
w=\psi=0 \quad \text { on } \partial \Omega .
$$

We shall not be concerned with other types of boundary conditions here. The existence of a unique solution of the problem described by (1.1) and (1.3) above for each $\varepsilon>0$ is established in [1]. We are interested in the behavior of the solutions as $\varepsilon \rightarrow 0$.

We observe that the system (1.1) is "singularly perturbed" with respect to $\varepsilon$ since the latter is multiplied to the highest-order derivatives in the equations. As $\varepsilon \rightarrow 0$, equations (1.1) imply

$$
\psi+\nabla w=0 ; \quad \nabla^{2} w+\nabla \cdot \psi=0
$$

Taking the divergence of (1.1a) and using (1.1b) yields

$$
\nabla^{4} w=\frac{p}{D},
$$

which is the biharmonic equation in the classical plate theory. From the first of (1.4) we have

$$
\psi_{x}=-\partial w / \partial x, \quad \psi_{y}=-\partial w / \partial y .
$$

Thus on the boundary $\partial \Omega$,

$$
w=-\partial w / \partial x=-\partial w / \partial y=0,
$$

which are the classical clamped plate boundary conditions. Denoting by $w_{0}$ the solution in the classical plate theory, we are led to expect, as $\varepsilon \rightarrow 0$,

$$
w \rightarrow w_{0}, \quad \psi_{x} \rightarrow-\partial w_{0} / \partial x, \quad \psi_{y} \rightarrow-\partial w_{0} / \partial y
$$

in some appropriate sense.

The above convergence was studied in [1]. More specifically, letting

$$
\begin{aligned}
& \mathbf{U}_{0}=\left(-\partial w_{0} / \partial x,-\partial w_{0} / \partial y, w_{0}\right), \\
& \mathbf{U}_{\varepsilon}=\left(\psi_{x}, \psi_{y}, w\right),
\end{aligned}
$$

we established in [1]

$$
\left\|\mathbf{U}_{\varepsilon}-\mathbf{U}_{0}\right\|_{1} \leqslant C \varepsilon^{1 / 2}\left\|\nabla\left(\nabla^{2} w_{0}\right)\right\|_{0},
$$

where $C$ is a constant, and the norms $\|\cdot\|_{0}$ and $\|\cdot\|_{1}$ above denote the appropriate norms in the Sobolev spaces $H^{0}(\Omega)$ and $\left(H^{1}(\Omega)\right)^{3}$ respectively. It was pointed out in [1] that for 
$p / D \in H^{0}(\Omega)$, the expression $\left\|\nabla\left(\nabla^{2} w_{0}\right)\right\|_{0}$ on the right of (1.10) is finite. Thus $\mathbf{U}_{\varepsilon} \rightarrow \mathbf{U}_{0}$ as $\varepsilon \rightarrow 0$ in the $\|\cdot\|_{1}$ norm. This also implies $\mathbf{U}_{\varepsilon} \rightarrow \mathbf{U}_{0}$ pointwise provided that these solutions have enough smoothness.

In this paper we are concerned with the detailed dependence of $\mathbf{U}_{\varepsilon}$ on $\varepsilon$ in the limit as $\varepsilon \rightarrow 0$. This is also related to the sharpness of the power $\varepsilon^{1 / 2}$ in (1.10) which is open to question. In particular, one would like to improve this power from $\varepsilon^{1 / 2}$ to $\varepsilon$, say, so that analyticity of $\mathbf{U}_{\varepsilon}$ at $\varepsilon=0$ might be concluded.

We shall show in the next section the nonexistence of regular asymptotic expansions of $\mathbf{U}_{\varepsilon}$ in integral powers of $\varepsilon$, ruling out the analyticity of $\mathbf{U}_{\varepsilon}$ at $\varepsilon=0$ except in special cases. In Section 3 we shall present an exact solution for a circular plate for which

$$
\left\|\mathbf{U}_{\varepsilon}-\mathbf{U}_{0}\right\|_{1}=O\left(\varepsilon^{3 / 4}\right) .
$$

The result in (1.11) shows that improvement of the power in (1.10) from $\varepsilon^{1 / 2}$ to $\varepsilon$ is not possible in general. This explicit solution also exhibits a boundary layer phenomenon near the clamped edge that decays rapidly away from the boundary.

2. The nonexistence of a regular asymptotic expansion for $\mathbf{U}_{\boldsymbol{\varepsilon}}$. For the boundary value problems in (1.1) and (1.3) let us seek solutions for $\psi$ and $w$ in the following form:

$$
\begin{aligned}
& \psi=\sum_{i=0}^{\infty} \varepsilon^{i} \psi_{i}, \\
& w=\sum_{i=0}^{\infty} \varepsilon^{i} w_{i},
\end{aligned}
$$

valid for small $\varepsilon$, with

$$
\psi_{i}=0, \quad w_{i}=0 \quad \text { on } \partial \Omega \text { for all } i .
$$

Substituting (2.1) into (1.1) and collecting like powers of $\varepsilon$ yields

$$
\begin{gathered}
\left\{\begin{array}{l}
\psi_{0}+\nabla w_{0}=0, \\
\nabla^{2} w_{0}+\nabla \cdot \psi_{0}=0,
\end{array}\right. \\
\left\{\begin{array}{l}
\psi_{1}+\nabla w_{1}=\frac{1}{2}\left[(1-\mu) \nabla^{2} \psi_{0}+(1+\mu) \nabla\left(\nabla \cdot \psi_{0}\right)\right], \\
\nabla^{2} w_{1}+\nabla \cdot \psi_{1}=-\frac{p}{D},
\end{array}\right. \\
\left\{\begin{array}{l}
\psi_{2}+\nabla w_{2}=\frac{1}{2}\left[(1-\mu) \nabla^{2} \psi_{1}+(1+\mu) \nabla\left(\nabla \cdot \psi_{1}\right)\right], \\
\nabla^{2} w_{2}+\nabla \cdot \psi_{2}=0,
\end{array}\right.
\end{gathered}
$$

Equations (2.3a) and (2.3b) are consistent but do not determine $\psi_{0}$ and $w_{0}$ at this order. Taking the divergence of (2.4a) and comparing with (2.4b) yields

$$
\nabla^{2}(\nabla \cdot \psi)=-\frac{p}{D}
$$


which upon using $(2.3 \mathrm{~b})$ becomes

$$
\nabla^{4} w_{0}=\frac{p}{D},
$$

showing $w_{0}$ is indeed the solution in the classical plate theory.

Next we take the divergence of (2.5a) and compare with (2.5b). This yields

$$
\nabla^{2}\left(\nabla \cdot \psi_{1}\right)=0
$$

Taking the Laplacian of (2.4b) and using (2.8) then leads to

$$
\nabla^{4} w_{1}=-\frac{1}{D} \nabla^{2} p
$$

However, we observe that the problem for $\psi_{1}$ and $w_{1}$ from (2.4) is overdetermined in general. To see this we rewrite (2.4a), with the aid of (2.3), as

$$
\psi_{1}+\nabla w_{1}=\nabla\left(\nabla^{2} w_{0}\right)
$$

On the boundary $\partial \Omega$, the left-hand side of $(2.10)$ as a vector is normal to $\partial \Omega$ since $\psi_{1}$ and $w_{1}$ both vanish there. However, the vector $\nabla\left(\nabla^{2} w_{0}\right)$ on $\partial \Omega$ in general will have both normal and tangential components. Thus $\psi_{1}$ and $w_{1}$ cannot meet the boundary conditions, and the regular asymptotic expansion breaks down.

We note that the tangential component of $\nabla\left(\nabla^{2} w_{0}\right)$ is related to the transverse shear force on vertical planes normal to the boundary. This tangential component of $\nabla\left(\nabla^{2} w_{0}\right)$ vanishes along $\partial \Omega$, for example, in the case of a circular plate under an axisymmetric loading.

On the other hand, the difficulty in meeting the boundary conditions by regular asymptotic expansions along with the singular perturbation nature of the governing equations suggests the possibility of the existence of boundary layer phenomena in the solutions. That this is indeed the case is studied in the next section.

3. Solutions for a clamped circular plate. We consider a clamped circular plate with unit radius. It is convenient to work in the polar coordinates $(r, \theta)$. To this end we write $\psi$ as

$$
\psi=\psi_{\mathbf{r}} \hat{\mathbf{r}}+\psi_{\theta} \hat{\theta}
$$

where $\psi_{r}$ and $\psi_{\theta}$ are the (negative of) plate rotations in the $r$ - and $\theta$-directions respectively. Equations (1.1) now become

$$
\begin{aligned}
\frac{\partial^{2} \psi_{r}}{\partial r^{2}}+\frac{1}{r} \frac{\partial \psi_{r}}{\partial r}+ & \frac{1}{2 r^{2}} \frac{\partial^{2} \psi_{r}}{\partial \theta^{2}}-\frac{1}{r^{2}} \psi_{r}-\frac{3}{2 r^{2}} \frac{\partial \psi_{\theta}}{\partial \theta}+\frac{1}{2 r} \frac{\partial^{2} \psi_{\theta}}{\partial r \partial \theta} \\
& +\mu\left(-\frac{1}{2 r^{2}} \frac{\partial^{2} \psi_{r}}{\partial \theta^{2}}+\frac{1}{2 r^{2}} \frac{\partial \psi_{\theta}}{\partial \theta}+\frac{1}{2 r} \frac{\partial^{2} \psi_{\theta}}{\partial r \partial \theta}\right)-\varepsilon^{-1}\left(\psi_{r}+\frac{\partial w}{\partial r}\right)=0
\end{aligned}
$$




$$
\begin{gathered}
\frac{1}{2} \frac{\partial^{2} \psi_{\theta}}{\partial r^{2}}+\frac{1}{2 r} \frac{\partial \psi_{\theta}}{\partial r}+\frac{1}{r^{2}} \frac{\partial^{2} \psi_{\theta}}{\partial \theta^{2}}-\frac{1}{2 r^{2}} \psi_{\theta}+\frac{3}{2 r^{2}} \frac{\partial \psi_{r}}{\partial \theta}+\frac{1}{2 r} \frac{\partial^{2} \psi_{r}}{\partial r \partial \theta}+ \\
\mu\left(-\frac{1}{2} \frac{\partial^{2} \psi_{\theta}}{\partial r^{2}}-\frac{1}{2 r} \frac{\partial \psi_{\theta}}{\partial r}+\frac{1}{2 r^{2}} \psi_{\theta}-\frac{1}{2 r^{2}} \frac{\partial \psi_{r}}{\partial \theta}+\frac{1}{2 r} \frac{\partial^{2} \psi_{r}}{\partial r \partial \theta}\right)-\varepsilon^{-1}\left(\psi_{\theta}+\frac{1}{r} \frac{\partial w}{\partial \theta}\right)=0 \\
\varepsilon^{-1}\left(\frac{\partial^{2} w}{\partial r^{2}}+\frac{1}{r} \frac{\partial w}{\partial r}+\frac{1}{r^{2}} \frac{\partial^{2} w}{\partial \theta^{2}}+\frac{\partial \psi_{r}}{\partial r}+\frac{1}{r} \psi_{r}+\frac{1}{r} \frac{\partial \psi_{\theta}}{\partial \theta}\right)=-\frac{p}{D}
\end{gathered}
$$

and the boundary conditions in (1.1) become

$$
w=\psi_{r}=\psi_{\theta}=0, \text { at } r=1 .
$$

For axisymmetric solutions $\psi_{\theta} \equiv 0$, and $\psi_{r}$ and $w$ are functions of $r$ only. The equations (3.1) degenerate into

$$
\begin{gathered}
\frac{d^{2} \psi_{r}}{d r^{2}}+\frac{1}{r} \frac{d \psi_{r}}{d r}-\frac{1}{r^{2}} \psi_{r}-\varepsilon^{-1}\left(\psi_{r}+\frac{d w}{d r}\right)=0 \\
\varepsilon^{-1}\left(\frac{d^{2} w}{d r^{2}}+\frac{1}{r} \frac{d w}{d r}+\frac{d \psi_{r}}{d r}+\frac{1}{r} \psi_{r}\right)=-\frac{p}{D}
\end{gathered}
$$

In the simplest case we take $p / D \equiv 1$. The boundary value problem above has the following solution:

$$
\begin{gathered}
w=\frac{1}{64}\left(1-r^{2}\right)^{2}+\frac{\varepsilon}{4}\left(1-r^{2}\right), \\
\psi_{r}=\frac{r}{16}\left(1-r^{2}\right) .
\end{gathered}
$$

We note that the corresponding problem in the classical plate theory for $w_{0}=w_{0}(r)$ :

$$
\begin{gathered}
\nabla^{4} w_{0}=1, \quad r<1 \\
w_{0}=\frac{d w_{0}}{d r}=0, \quad \text { at } r=1,
\end{gathered}
$$

has the solution

$$
w_{0}=\frac{1}{64}\left(1-r^{2}\right)^{2}
$$

Thus

$$
\begin{gathered}
w=w_{0}+\frac{\varepsilon}{4}\left(1-r^{2}\right), \\
\psi_{r}=-\frac{d w_{0}}{d r},
\end{gathered}
$$

and the difference $\mathbf{U}_{\boldsymbol{\varepsilon}}-\mathbf{U}_{0}$ involves integral powers of $\varepsilon$ only. The same is to be expected for more general axisymmetric load $p=p(r)$, but we shall not pursue this here further.

We now consider the nonaxisymmetric load $p / D=\cos \theta$. For this case the solution in the classical plate theory may be obtained by assuming a form $w_{0}=R(r) \cos \theta$. This 
separation of variables leads to

$$
\begin{aligned}
w_{0} & =\frac{r}{90}(1-r)^{2}(2 r+1) \cos \theta, \\
\frac{\partial w_{0}}{\partial r} & =-\frac{1}{90}(1-r)\left(8 r^{2}-r-1\right) \cos \theta, \\
\frac{\partial w_{0}}{\partial \theta} & =-\frac{r}{90}(1-r)^{2}(2 r+1) \sin \theta .
\end{aligned}
$$

With $p / D=\cos \theta$, the solutions of the problem described by (3.2) and (3.3) are rather complicated. We present these solutions below with their detailed derivations given in the Appendix:

$$
\begin{gathered}
w=\left\{\frac{r}{90}(1-r)^{2}(2 r+1)+\varepsilon r(1-r)\left(\tilde{a}(r+1)-\frac{r}{3}\right)\right\} \cos \theta, \\
\psi_{r}+\frac{\partial w}{\partial r}=\left\{\varepsilon\left(\frac{11-30 \tilde{a}}{15 r} \frac{I_{1}(\alpha r)}{I_{1}(\alpha)}-\frac{2 r}{3}+\frac{4}{15}\right)\right. \\
\left.\quad+\frac{8 \varepsilon^{2}}{3}\left(\frac{-3 \tilde{a}+1}{r} \frac{I_{1}(\alpha r)}{I_{1}(\alpha)}-1+3 \tilde{a}\right)\right\} \cos \theta, \\
\psi_{\theta}+\frac{1}{r} \frac{\partial w}{\partial \theta}=\left\{\varepsilon^{1 / 2} \frac{\sqrt{2}}{\sqrt{1-\mu}} \frac{30 \tilde{a}-11}{15} \frac{I_{0}(\alpha r)}{I_{1}(\alpha)}\right. \\
-\varepsilon\left(\frac{30 \tilde{a}-11}{15 r} \frac{I_{1}(\alpha r)}{I_{1}(\alpha)}-\frac{5 r-4}{15}\right) \\
\quad-\frac{8}{3}(1-3 \tilde{a}) \varepsilon^{3 / 2} \frac{\sqrt{2}}{\sqrt{1-\mu}} \frac{I_{0}(\alpha r)}{I_{1}(\alpha)} \\
\left.+\frac{8}{3}(1-3 \tilde{a}) \varepsilon^{2}\left(\frac{I_{1}(\alpha r)}{r I_{1}(\alpha)}+1\right)\right\} \sin \theta
\end{gathered}
$$

where

$$
\begin{gathered}
\alpha=\frac{\sqrt{2}}{\sqrt{1-\mu}} \varepsilon^{-1 / 2}, \\
\tilde{a}=\frac{1}{\varepsilon} \frac{-\frac{4}{5} \alpha^{-1} \varepsilon-\frac{16}{3} \alpha^{-1} \varepsilon^{2}+\frac{I_{0}(\alpha)}{I_{1}(\alpha)}\left(\frac{11}{15} \varepsilon+\frac{8}{3} \varepsilon^{2}\right)}{(2+8 \varepsilon) \frac{I_{0}(\alpha)}{I_{1}(\alpha)}-2 \alpha^{-1}-16 \alpha^{-1} \varepsilon},
\end{gathered}
$$

and $I_{0}$ and $I_{1}$ denote modified Bessel functions [4].

We note that the solutions in (3.11) also depend on $\varepsilon$ through $\tilde{a}$ and $\alpha$. When $\varepsilon$ is small, $\alpha$ is large. Expansions of Bessel functions for large arguments are known:

$$
I_{\nu}(x) \sim \frac{e^{x}}{\sqrt{2 \pi x}}\left[1-\frac{4 \nu^{2}-1}{8 x}+\frac{\left(4 \nu^{2}-1\right)\left(4 \nu^{2}-9\right)}{2 !(8 x)^{2}}+\cdots\right] \text {. }
$$


From this we obtain

$$
\frac{I_{0}(x)}{I_{1}(x)} \sim 1+\frac{1}{2 x}+\frac{3}{8 x^{2}}+O\left(\frac{1}{x^{3}}\right) .
$$

Using (3.12a) along with (3.14) in (3.12b) then yields

$$
\tilde{a}=\frac{1}{30}\left[11-\varepsilon^{1 / 2} \frac{\sqrt{1-\mu}}{\sqrt{2}}-\varepsilon\left(88+\frac{37(1-\mu)}{8}\right)\right]+O\left(\varepsilon^{3 / 2}\right) .
$$

With the help of (3.15) and (3.10a) we can rewrite (3.11a) as

$$
w=w_{0}+\frac{\varepsilon r}{2}(1-r)(r+11)+O\left(\varepsilon^{3 / 2}\right) .
$$

Likewise, with the help of (3.10b), (3.10c), and (3.16a) we can rewrite (3.11b) and (3.11c) as

$$
\begin{aligned}
\psi_{r}= & -\frac{\partial w_{0}}{\partial r}-\frac{\varepsilon}{10}\left(1-r^{2}\right) \cos \theta \\
& +\frac{\varepsilon^{3 / 2} \sqrt{1-\mu}}{15 \sqrt{2}} \frac{I_{1}\left(\frac{\sqrt{2}}{\sqrt{1-\mu}} \varepsilon^{-1 / 2} r\right)}{r I_{1}\left(\frac{\sqrt{2}}{\sqrt{1-\mu}} \varepsilon^{-1 / 2}\right)} \cos \theta+O\left(\varepsilon^{3 / 2}\right), \\
\psi_{\theta}= & -\frac{1}{r} \frac{\partial w_{0}}{\partial \theta}-\frac{\varepsilon}{15} \frac{I_{0}\left(\frac{\sqrt{2}}{\sqrt{1-\mu}} \varepsilon^{-1 / 2} r\right)}{I_{1}\left(\frac{\sqrt{2}}{\sqrt{1-\mu}} \varepsilon^{-1 / 2}\right)} \sin \theta \\
& +\frac{\varepsilon}{30}\left(3-r^{2}\right) \sin \theta+O\left(\varepsilon^{3 / 2}\right) .
\end{aligned}
$$

It is seen from the above that the leading terms in $w-w_{0}, \psi_{r}+\partial w_{0} / \partial r$, and $\psi_{\theta}+(1 / r)\left(\partial w_{0} / \partial \theta\right)$ are of the order $O(\varepsilon)$. However, this is not the case of the radial derivative of (3.16c). More specifically, using the identity $I_{0}^{\prime}(x)=I_{1}(x)$ we obtain from (3.16c)

$$
\frac{\partial}{\partial r}\left(\psi_{\theta}+\frac{1}{r} \frac{\partial w_{0}}{\partial \theta}\right)=-\frac{\varepsilon^{1 / 2} \sqrt{2}}{15 \sqrt{1-\mu}} \frac{I_{1}\left(\frac{\sqrt{2}}{\sqrt{1-\mu}} \varepsilon^{-1 / 2} r\right)}{I_{1}\left(\frac{\sqrt{2}}{\sqrt{1-\mu}} \varepsilon^{-1 / 2}\right)} \sin \theta+O(\varepsilon),
$$

and the first term on the right is of the order $O\left(\varepsilon^{1 / 2}\right)$. This can be seen by using (3.13) to expand the ratio of the Bessel functions for $r$ bounded away from zero, resulting in

$$
\frac{I_{1}(\alpha r)}{I_{1}(\alpha)} \sim \frac{e^{-\alpha(1-r)}}{r^{1 / 2}}\left(1-\frac{3(1-r)}{8 \alpha r}-\frac{3(1-r)(11 r+5)}{128 \alpha^{2} r^{2}}+\cdots\right) .
$$


This indicates that for large $\alpha$

$$
\frac{I_{1}\left(\frac{\sqrt{2}}{\sqrt{1-\mu}} \varepsilon^{-1 / 2} r\right)}{I_{1}\left(\frac{\sqrt{2}}{\sqrt{1-\mu}} \varepsilon^{-1 / 2}\right)} \sim \frac{\exp \left\{-\frac{\sqrt{2}}{\sqrt{1-\mu}} \varepsilon^{-1 / 2}(1-r)\right\}}{r^{1 / 2}}=O\left(\varepsilon^{0}\right),
$$

and indeed the first term on the right of (3.17) is the dominant term for small $\varepsilon$. Furthermore, the result above shows this dominant term decays exponentially as $r$ moves away from the boundary and is significant only over a region of length $O\left(\varepsilon^{1 / 2}\right)$ near $r=1$. Based on these considerations and carrying out the necessary differentiations in Cartesian coordinates, it is easy to verify that

as we stated in (1.11).

$$
\left\|\mathbf{U}_{\varepsilon}-\mathbf{U}_{0}\right\|_{1}=O\left(\varepsilon^{3 / 4}\right)
$$

4. Discussions and concluding remarks. There exist various plate theories in the engineering mechanics literature resulting from the theory of elasticity using different approximation schemes. This paper concerns two such theories: the classical plate theory that neglects shear deformation and the Reissner-Mindlin plate theory in which shear deformation is incorporated. Some general discussions on the relative smoothness of solutions of the governing equations in these two theories, without the consideration of boundary conditions, are given in [5]. Our equation (A.1) in the Appendix also reveals some of the same information. Our primary interest, however, is in the properties of solutions of boundary value problems under clamped edge conditions.

It is seen in the Introduction that a small parameter $\varepsilon$ may be introduced in the Reissner-Mindlin theory such that as $\varepsilon \rightarrow 0$ the governing equations go over to the biharmonic equation of the classical plate theory. From equation (1.2) it is seen that the limit $\varepsilon \rightarrow 0$ may be achieved either by letting $G^{\prime}\left(\right.$ or $\left.\kappa^{2}\right) \rightarrow \infty$, thereby making the plate infinitely rigid with respect to shear deformation, or by letting $h_{0} \rightarrow 0$, i.e., by making the plate infinitely thin. It should be borne in mind in the latter case, however, that the plate rigidity $D$ also depends on $h_{0}$ and provisions must be made so that $D$ remains finite (not going to zero) and the plate does not become a membrane.

We may refer to the mathematical problem for $\varepsilon>0$ as the $P_{\varepsilon}$-problem and the corresponding problem in the classical plate theory as the $P_{0}$-problem. We are specifically interested in the manner in which solutions of the $P_{\varepsilon}$-problem tend to those of the $P_{0}$-problem. The estimate given in (1.10) that was derived in [1] shows this convergence in the $\|\cdot\|_{1}$ norm but leaves the question of the detailed dependence of the solutions of the $P_{\varepsilon}$-problems on $\varepsilon$ as $\varepsilon \rightarrow 0$ unanswered. Our results in Sections 2 and 3 above, which we alluded to in [1], suggest that such dependence on $\varepsilon$ as $\varepsilon \rightarrow 0$ indeed may be rather complicated.

It is interesting to note that our investigation in [1] was motivated by an earlier work by Westbrook [6], who arrived at the same Reissner-Mindlin equations as the basis for justifying a penalty function finite element method for treating clamped plate problems in the classical plate theory. We have also pursued the analysis of this penalty function finite element method in [7]. Because of the singular perturbation nature of the $P_{\varepsilon}$-problem, 
accurate numerical solutions for small $\varepsilon$ require the use of small mesh size $h$. Due to the limitations on the use of arbitrarily small mesh sizes $h$ on the computers some extrapolations to $\varepsilon=0$ become inevitable. The success of the extrapolations depends heavily on our knowledge of how the solutions depend on $\varepsilon$ as $\varepsilon \rightarrow 0$, as illustrated by the example in [7].

We have seen in Section 2 that the nonvanishing of the tangential component of $\nabla\left(\nabla^{2} w_{0}\right)$ on the boundary prevented the construction of regular asymptotic series solution for the $P_{\varepsilon}$-problem. We mention also that both the dependence on fractional powers of $\varepsilon$ and the boundary layer phenomenon in Section 3 for the circular plate under the load $p / D=\cos \theta$ are associated with the radial derivative of $\psi_{\theta}+(1 / r)\left(\partial w_{0} / \partial r\right)$. In particular, $\partial \psi_{\theta} / \partial r$ is related to the twisting moment in the plate. It appears that for all geometries of clamped plates, and for all loads, with the exception of a circular plate with axisymmetric load, some twisting moment is expected, along with transverse shear force in vertical planes perpendicular to the boundary. Thus boundary layer phenomena are inevitable in the solutions of such $P_{\varepsilon}$-problems.

It is known (see [8] and the references contained therein) that an asymptotic sequence of equations, in terms of a small thickness parameter, may be developed from the equations of elastostatics. The leading term in this sequence is the classical plate theory. There lies the difficulty, however, in deriving appropriate boundary conditions for the various asymptotic plate theories. Gregory and Wan in [8] have recently devised a general scheme, based on an application of the Betti-Rayleigh reciprocal theorem, that enables the derivation of correct boundary conditions for the classical as well as the higher-order plate theories for general admissible edge data. They considered in [8], in particular, the problem of axisymmetric bending of a simply-supported circular plate. The results in [8] seem to suggest that the $\varepsilon^{1 / 2}$ dependence even occurs in the axisymmetric case as the true plate solution approaches the classical plate solution for simply-supported edges and similar conclusions may hold for other types of boundary conditions. If this is indeed the case our results obtained here would be in variance with those in [8] and this would rule out the Reissner-Mindlin theory being a legitimate asymptotic plate theory. A detailed comparison based on the respective explicit solutions is beyond the scope of the paper.

Acknowledgment. We wish to thank the referee for bringing Reference [8] to our attention.

Appendix. Derivations of the solutions in (3.11). To find solutions for $\psi_{r}, \psi_{\theta}$, and $w$, with $p / D=\cos \theta$, of equations (3.2) it is useful to observe that it is possible to eliminate $\psi$ from (1.1a) and (1.1b) and obtain

$$
\nabla^{4} w=\frac{p}{D}-\varepsilon \nabla^{2} \frac{p}{D}
$$

We seek solution for $w$, in polar coordinates, in the form

$$
w=r C(r) \cos \theta,
$$

along with the boundary condition $w=0$ at $r=1$. This results in

$$
C(r)=(r-1)\left[-a(r+1)+\frac{1}{90}(r-1)(2 r+1)+\frac{\varepsilon}{3} r\right],
$$

where $a$ is an undetermined constant since only one boundary condition at $r=1$ is given. 
We substitute this $w$ into (3.2), make the changes of dependent variables,

$$
\begin{aligned}
& \xi \cos \theta=\psi_{r}+\frac{\partial w}{\partial r}, \\
& \eta \sin \theta=\psi_{\theta}+\frac{1}{r} \frac{\partial w}{\partial \theta}
\end{aligned}
$$

and obtain the following set of ordinary differential equations:

$$
\begin{gathered}
r^{2} \xi^{\prime \prime}+r \xi^{\prime}+\left(-\frac{3}{2}+\frac{\mu}{2}-\varepsilon^{-1} r^{2}\right) \xi+\frac{1+\mu}{2} r \eta^{\prime}+\frac{\mu-3}{2} \eta \\
=r^{2}\left(C+r C^{\prime}\right)^{\prime \prime}+r\left(C+r C^{\prime}\right)^{\prime}-2 r C^{\prime}, \\
\frac{1-\mu}{2} r^{2} \eta^{\prime \prime}+\frac{1-\mu}{2} r \eta^{\prime}+\left(\frac{\mu-3}{2}-\varepsilon^{-1} r^{2}\right) \eta-\frac{1+\mu}{2} r \xi^{\prime}+\frac{\mu-3}{2} \xi \\
=-r^{2} C^{\prime \prime}-3 r C^{\prime}, \\
r \xi^{\prime}+\xi+\eta=-\varepsilon r .
\end{gathered}
$$

From (A.5c) we have

$$
\eta=-r \xi^{\prime}-\xi-\varepsilon r
$$

Eq. (A.5a) thus reduces to

$$
\begin{aligned}
& r^{2} \xi^{\prime \prime}+3 r \xi^{\prime}-\frac{2 \varepsilon^{-1} r^{2}}{1-\mu} \xi \\
& \quad=\frac{2}{1-\mu}\left[r^{2}\left(C+r C^{\prime}\right)^{\prime \prime}+r\left(C+r C^{\prime}\right)^{\prime}-2 r C^{\prime}-(1-\mu) \varepsilon r\right] .
\end{aligned}
$$

We set

$$
P=r \xi
$$

and

$$
\rho=\alpha r
$$

where $\alpha=\sqrt{2} \varepsilon^{-1 / 2} / \sqrt{1-\mu}$, and regard $P$ as a function of $\rho$. Eq. (A.7) then becomes the nonhomogeneous Bessel equation

$$
\begin{aligned}
\rho^{2} P^{\prime \prime}+\rho P^{\prime}- & \left(1+\rho^{2}\right) P \\
= & \varepsilon \rho^{2}\left[\frac{1-\mu}{3} \varepsilon \rho^{2}+8\left(-a-\frac{1}{30}+\frac{\varepsilon}{3}\right) \sqrt{\frac{1-\mu}{2}} \varepsilon^{1 / 2} \rho-(1-\mu) \varepsilon\right] .
\end{aligned}
$$

A general solution to (A.10) is given as a general solution to the homogeneous equation, $P_{\text {HOM }}$, plus a particular solution, $P_{\text {PART }}$,

$$
P=P_{\mathrm{HOM}}+P_{\mathrm{PART}} .
$$

$P_{\text {HOM }}$ is of the form

$$
P_{\text {HOM }}=A I_{1}(\rho)+B K_{1}(\rho) \text {, }
$$


where $A$ and $B$ are arbitrary constants and $I_{1}$ and $K_{1}$ are modified Bessel functions. A particular solution is

$$
P_{\mathrm{PART}}=-\frac{2}{3} \alpha^{-2} \varepsilon \rho^{2}-8\left(-a-\frac{1}{30}+\frac{\varepsilon}{3}\right) \alpha^{-1} \varepsilon \rho .
$$

Thus

$$
\xi(r)=\frac{1}{r}\left[A I_{1}(\alpha r)+B K_{1}(\alpha r)+P_{\mathrm{PART}}\right] .
$$

Since $(1 / r) K_{1}(\alpha r)$ is singular at the origin we must set $B=0$. Next by the condition $\psi_{r}=0$ at $r=1$, it follows from (A.4a) that

$$
\xi(1)=-2 a+\frac{\varepsilon}{3}
$$

Thus from (A.13) we have

$$
A=\frac{-8 a \varepsilon+\frac{11}{15} \varepsilon+\frac{8}{3} \varepsilon^{2}-2 a}{I_{1}(\alpha)}
$$

We can now substitute $\xi(r)$ into (A.6) to determine $\eta(r)$, where the condition $\psi_{\theta}=0$, at $r=1$ serves to determine the constant $a$. The solutions for $\xi(r)$ and $\eta(r)$ are then to be substituted into (A.5b) to ensure all three equations (A.5) are satisfied. Now with $\psi_{\theta}=0$ at $r=1$ we have $\eta(1)=0$. From (A.6) it follows that

$$
0=\frac{\varepsilon}{3}-\left(-2 a-8 a \varepsilon+\frac{11}{15} \varepsilon+\frac{8}{3} \varepsilon^{2}\right) \alpha \frac{I_{1}^{\prime}(\alpha)}{I_{1}(\alpha)}+8 \varepsilon\left(-a-\frac{1}{30}+\frac{\varepsilon}{3}\right) .
$$

Using the Bessel function identity

$$
I_{1}^{\prime}(x)=I_{0}(x)-\frac{1}{x} I_{1}(x)
$$

and solving for $a$ from (A.17) we finally have

$$
a=\frac{-\frac{4}{5} \alpha^{-1} \varepsilon-\frac{16}{3} \alpha^{-1} \varepsilon^{2}+\frac{I_{0}(\alpha)}{I_{1}(\alpha)}\left(\frac{11}{15} \varepsilon+\frac{8}{3} \varepsilon^{2}\right)}{(2+8 \varepsilon) \frac{I_{0}(\alpha)}{I_{1}(\alpha)}-2 \alpha^{-1}-16 \alpha^{-1} \varepsilon}
$$

Now defining $\tilde{a}$ by

$$
\tilde{a}=\varepsilon^{-1} a,
$$

we obtain the solutions for $\psi_{r}, \psi_{\theta}$, and $w$ given in (3.11).

\section{REFERENCES}

[1] T. C. Assiff and D. H. Y. Yen, On a penalty-perturbation theory for plate problems, IMA J. Appl. Math. 34, 121-136 (1985)

[2] E. Reissner, The effect of transverse shear deformation on the bending of elastic plates, J. Appl. Mech. 12, Trans. ASME 67, A-69-A-77, 7-42 (1945)

[3] R. D. Mindlin, Influence of ratatory inertia and shear on flexural motions of isotropic elastic plates, J. Appl. Mech. 18, 31-38 (1951)

[4] M. Abramowitz and I. A. Stegun, Handbook of Mathematical Functions, Dover Inc., New York (1965) 
[5] B. Fraeijs de Veubeke, A Course in Elasticity, Springer-Verlag, New York (1979)

[6] D. R. Westbrook, A variational principle with applications in finite elements, J. Inst. Math. Applics. 14, 79-82 (1974)

[7] T. C. Assiff and D. H. Y. Yen, On a penalty-perturbation finite element method for boundary value problems for elastic plates, Proceedings of the International Conference on Accuracy Estimates and Adaptive Refinements in Finite Element Computations, Lisbon, Portugal 1, 189-198 (1984)

[8] R. D. Gregory and F. Y. M. Wan, On plate theories and Saint-Venant's principle, Internat. J. Solids Structures 21, 1005-1024 (1985) 